\title{
REVIEW ARTICLE \\ ON THE MATLAB TECHNIQUE BY USING LAPLACE TRANSFORM FOR SOLVING SECOND ORDER ODE WITH INITIAL CONDITIONS EXACTLY
}

\author{
Bawar Mohammed Faraj* and Faraedoon Waly Ahmed \\ Department of Physics, College of Science, University of Halabja, Halabja, Iraq \\ *Corresponding Author E-mail: bawarm.faraj@uoh.edu.iq
}

This is an open access article distributed under the Creative Commons Attribution License, which permits unrestricted use, distribution, and reproduction in any medium, provided the original work is properly cited.

\section{ARTICLE DETAILS}

\section{Article History:}

Received 10 June 2019 Accepted 15 July 2019 Available online 1 August 2019

\section{ABSTRACT}

In this paper Matlab technique has been presented that is approach to exact solution for second order ODE with constant coefficients and initial condition by using Laplace transformation. Matlab function has been constructed to estimate and compute exact solution of second order ordinary differential equations with initial conditions generally, the results of the program shows the elapsed time, exact solution and it's figures.

\section{KEYWORDS}

Ordinary differential equation, Matlab program, Laplace transform, Initial value problems.

\section{INTRODUCTION}

Most ordinary differential equations arising in science and engineering applications cannot be solved easily or it takes too much time, Classical methods may not be able to give solution. A transformations method can be used to get an exact solution to a differential equation. There are many methods which use transformation, Laplace transforms are commonly used in solving mathematical and physical problems which contains ordinary or partial differential equations with constant coefficients and integral equations as those problems are arising in the many branches of physics as electronic circuit analysis [1-15]. But it takes too much time for solving by hand and in papers, These ODE can be analyzed. However, mathematical software's was developed for solving the ordinary differential equations easily in some seconds, To compute quickly the solution of differential equation, One of such powerful and must know software's language is MATLAB language, which is easy to use, developed by Mathworks, Matlab program is contains Many easy to use tools, programs and functions to solve or simulate differential equation by Laplace transform and numerical methods such [16-19]. In last decades must of researcher developed new functions and programs for Matlab program for solving and computing exact solution for ordinary differential equations, developed functions estimated exact solution in some second with graph of exact solution, which reduced too many time for mathematicians and physicist in solving these problem by hand in paper, in this paper we developed a Matlab function by using Laplace transformation for estimating exact solution and it's graph in less than one percentage of a second, this function is general for every second order ordinary differential equations with constant coefficient and initial conditions, which has continuous $f(t)$ and satisfied Laplace's condition [20].

In this paper, we consider second order ordinary differential equation with constant coefficients and initial conditions.

$$
\left\{\begin{array}{l}
a u_{t t}+b u_{t}+c u=f(t), \\
u(0)=\varphi(t), u_{t}(0)=\psi(t),
\end{array}\right.
$$

where $a, b, c$ be constant coefficients, above ordinary differential equations can be classify to Hyperbolic, if $b^{2}-4 a c>0$, Parabolic, if $b^{2}-$ $4 a c=0$ and Elliptic, if $b^{2}-4 a c<0$.
Definition 1.1 Let $f(t)$ be a function of $t$ defined $\forall t>0$. Then the Laplace Transform of $f(t)$ denoted by $F(s)=\mathcal{L}\{f(t)\}$ is defined by

$$
F(s)=\mathcal{L}\{f(t)\}=\int_{0}^{\infty} e^{-s t} f(t) d t
$$

where $=a+i b, a, b \in \Re$, the Laplace transform has a linearity property. [2]

Apply the definition for first and second derivatives, we get

$$
\begin{array}{r}
\mathcal{L}\left\{u_{t t}(t)\right\}=s^{2} U(s)-s u(0)-u_{t}(0), \mathcal{L}\left\{u_{t}(t)\right\}=s U(s)- \\
u(0), \quad \mathcal{L}\{u(t)\}=U(s) .
\end{array}
$$

In this paper we used initial conditions with ODE.

Definition 1.2 Initial conditions (initial value problem) A differential equation with subsidiary conditions on the unknown function and its derivatives, which all given at the same value of the independent variable, the problem is called initial value problem, and the subsidiary conditions are called initial conditions [21-26].

Method to solve linear ordinary differential (ODE) by using Laplace transformation has the advantage of using the Laplace transform is that it yields the Particular solutions directly without the necessity of first finding the general solution and then evaluating the arbitrary constants. Laplace transformation can be apply for Ordinary and Partial differential equation by using this steps, Firstly, Start with differential equation with initial condition. Then Take Laplace Transform on both sides of equation and obtain subsidiary equation. Finally Take inverse of Laplace transformation and simplify it.

Example 1.1 Consider the following initial value problem

$$
\left\{\begin{array}{l}
u_{t t}+3 u_{t}+2 u=(\cos (t)-\sin (t)) e^{-t} \\
u(0)=0, u_{t}(0)=1
\end{array}\right.
$$

We have $u_{t t}+3 u_{t}+2 u=(\cos t-\sin t) e^{-t}$, take Laplace transform for both sides

$$
\mathcal{L}\left\{u_{t t}\right\}+3 \mathcal{L}\left\{u_{t}\right\}+2 \mathcal{L}\{u\}=\mathcal{L}\left\{\cos (t) e^{-t}-\sin (t) e^{-t}\right\},
$$

using the rules and for derivatives in definition (1.1), where $U(s)=\mathcal{L}\{u\}$, 
we obtain

$$
\begin{array}{r}
s^{2} U(s)-s u(0)-u_{t}(0)+3(s U(s)-u(0))+2 U(s) \\
=\frac{s+1}{(s+1)^{2}+1}-\frac{1}{(s+1)^{2}+1},
\end{array}
$$

Substituting initial conditions,

$$
s^{2} U(s)-1+3 s U(s)+2 U(s)=\frac{s}{(s+1)^{2}+1}
$$

which can be written

$$
\left(s^{2}+3 s+2\right) U(s)=\frac{s}{(s+1)^{2}+1}+1,
$$

from it we obtain

$$
U(s)=\frac{\frac{s}{(s+1)^{2}+1}+1}{\left(s^{2}+3 s+2\right)}
$$

simplifying it, yields

$$
U(s)=\frac{1}{(s+1)^{2}+1} .
$$

Take Laplace inverse for both sides

$$
u(t)=\mathcal{L}^{-1}\{U(s)\}=\mathcal{L}^{-1}\left\{\frac{1}{(s+1)^{2}+1}\right\}=\sin (t) e^{-t} .
$$

The exact solution for problem (3) is $(t)=\sin (t) e^{-t}$.

\section{MATLAB TECHNIQUE}

We consider initial value problem (1), and definition of Laplace transform (1.1) the Matlab function is presented, in this program $a, b, c$ are constant coefficients of ODE, $u 0$ is $u(0)$ and ut 0 is $u_{t}(0)$ initial conditions, function $f(t)$ must be input after calling the matlplace.m function. $f(t)$ it must be input as the independent variable $t$. Matlab Technique are defined as follow in Matlab's editor window

function $[\mathrm{a}, \mathrm{b}, \mathrm{c}, \mathrm{u} 0, \mathrm{ut} 0]=$ matlaplace $(\mathrm{a}, \mathrm{b}, \mathrm{c}, \mathrm{u} 0, \mathrm{ut} 0)$

syms s t U

$\mathrm{f}=$ input('input function $\mathrm{f}=$ ');

tic

$\mathrm{F}=$ laplace $(\mathrm{f}, \mathrm{t}, \mathrm{s})$;

$\mathrm{Ut}=\mathrm{s} * \mathrm{U}+\mathrm{u} 0 ; \mathrm{Utt}=\mathrm{s} * \mathrm{Ut}-\mathrm{ut} 0$;

Sol = solve $\left(a^{*} U t t+b^{*} U t+c^{*} U-F, U\right)$;

disp('The exact solution by Laplace transfromation is ')

$\mathrm{u}=\mathrm{ilaplace}(\mathrm{Sol}, \mathrm{s}, \mathrm{t})$

toc

ezplot(u)

grid on

title('Figure of Exact Solution by Laplace Transform')

xlabel('t'),ylabel('u(t)')

end

saving the program in Matlabs current folder and calling it, it will find exact solution and generate the graph and elapsed time for it. Back to example (1.1).

>> matlaplace $(1,3,2,0,1)$

input function $\mathrm{f}=(\cos (\mathrm{t})-\sin (\mathrm{t}))^{*} \exp (-\mathrm{t})$

The exact solution by Laplace transformation is

$\mathrm{u}=\exp (-\mathrm{t})^{*} \sin (\mathrm{t})$

Elapsed time is 0.050639 seconds

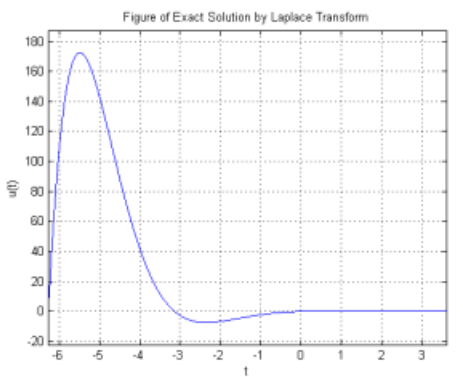

Figure 1: Figure of exact solution of (3)
Consider another initial value problem

$$
\left\{\begin{array}{l}
u_{t t}+u=\sin (t) \cos (t)+\frac{e^{t}}{\csc (t)}-t^{2}, \\
u(0)=0, u_{t}(0)=1,
\end{array}\right.
$$

which takes too much time for finding exact solution in another programs or by hand and paper, but matlaplace function gives a solution and it is figure in seconds.

>> matlaplace $(1,0,1,0,1)$

input function $\mathrm{f}=\sin (\mathrm{t}) * \cos (\mathrm{t})+\exp (\mathrm{t}) / \csc (\mathrm{t})-\mathrm{t}^{\wedge} 2$

The exact solution by Laplace transformation is $\mathrm{u}=\left(23^{*} \sin (\mathrm{t})\right) / 15-\left(8^{*} \cos (\mathrm{t})\right) / 5-\sin \left(2^{*} \mathrm{t}\right) / 6-\left(2^{*} \exp (\mathrm{t}) *(\cos (\mathrm{t})\right.$ $\sin (\mathrm{t}) / 2)) / 5-\mathrm{t}^{\wedge} 2+2$

Elapsed time is 0.055041 seconds.

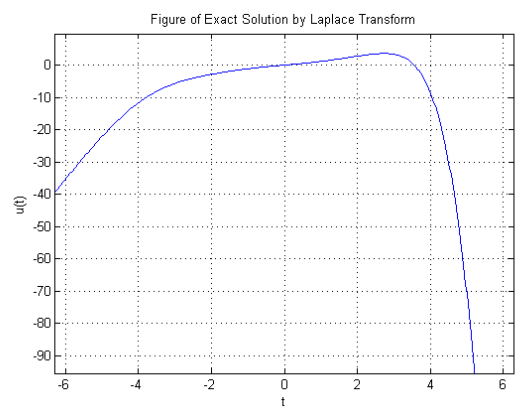

Figure 2: Figure of exact solution of (4)

\section{CONCLUSIONS}

Matlab software saves and reduces a lot of time in routine calculations and application for mathematicians, physicist, engineers and scientists. In this work Laplace transform are defined and applied for solving an ODE example classically, Matlab program are used to programming Laplace transformation for initial value problem, second order ordinary differential equations with constant coefficient, MATLAB function are constructed, estimates exact solution in less than a second, which makes easy and useful for researcher, and generates the graph of exact solution. Matlab program is possible to simulate the Laplace transformable equations directly which has made a good advancement in the research field.

\section{REFERENCES}

[1] Schiff, J.L. 1999. The Laplace Transform: Theory and Applications, Springer Science and Business Media.

[2] Akgul, A. 2015. New reproducing kernel functions. Mathematical Problems in Engineering.

[3] Inc, M., Akgul, A., Kilicman, A. 2013. Numerical solutions of the secondorder one dimensional telegraph equation based on reproducing kernel Hilbert space method. In Abstract and Applied Analysis, Hindawi.

[4] Akgul, A., Khan, Y., Akgul, E.K., Baleanu, D., Al Qurashi, M.M. 2017. Solutions of nonlinear systems by reproducing kernel method. The Journal of Nonlinear Sciences and Applications, 10, 4408-4417.

[5] Inc, M., Akgul, A., Kilicman, A. 2013. A novel method for solving KdV equation based on reproducing kernel Hilbert space method. In Abstract and Applied Analysis, Hindawi.

[6] Hashemi, M.S., Inc, M., Kilic, B., Akgul, A. 2016. On solitons and invariant solutions of the Magneto-electro-elastic circular rod. Waves in Random and Complex Media, 26(3), 259-271.

[7] Akgul, A., Kilicman, A., Baleanu, D. 2016. A new approach for onedimensional sine-Gordon equation. Advances in Difference Equations, (1), 8.

[8] Bawar F., Mahmut, M. 2018. Using Difference Scheme Method for the Numerical Solution of Telegraph Partial Differential Equation. Journal of Garmian University, 4, 157-163. 10.13140/RG.2.2.13922.99524.

[9] Bawar, F., Daban, D. 2018. Using matlab-simulink environment for an 
agricultural greenhouse. International IFS and Contemporary Mathematics Conference, 9-9. doi:10.13140/RG.2.2.25943.52646.

[10] Tchier, F., Inc, M., Kilic, B., Akgül, A. 2017. On soliton structures of generalized resonance equation with time dependent coefficients. Optik, $128,218-223$.

[11] Akgül, A., Hashemi, M.S., Raheem, S.A. 2017. Constructing two powerful methods to solve the Thomas-Fermi equation. Nonlinear Dynamics, 87(2), 1435-1444.

[12] Boutarfa, B., Akgül, A., Inc, M. 2017. New approach for the FornbergWhitham type equations. Journal of Computational and Applied Mathematics, 312, 13-26.

[13] Akgul, A., Kilicman, A. 2015. Solving delay differential equations by an accurate method with interpolation. In Abstract and Applied Analysis, Hindawi.

[14] Akgül, A., Baleanu, D. 2017. On solutions of variable-order fractional differential equations. An International Journal of Optimization and Control: Theories \& Applications (IJOCTA), 7(1), 112-116.

[15] Abramowitz, M., Stegun, I.A. 1965. Handbook of mathematical functions with formulas, graphs, and mathematical table. New York: Dover, 2172.

[16] Legua, M.P. 2006. The heaviside function and Laplace transforms, Proceedings of the $10^{\text {th }}$ WSEAS International Conference on Applied Mathematics, USA, 445-450.

[17] Faraj, B., Mondali, M. 2017. Using Difference Scheme Method for the Numerical Solution of Telegraph Partial Differential Equation. Journal of
Garmian University, 4(ICBS Conference), 157-163. doi: 10.24271/garmian.133

[18] Symbolic Math Toolbox User's Guide. 2006. The MathWorks, Inc. (www.mathworks.com).

[19] Kadry, S. 2014. Learning basic mathematics using MATLAB, International Journal of Information Technology \& Computer Science, 14(A202), 48-56.

[20] Rai, P. 2015. Applications of Laplace Transform to solve ODE Using MATLAB, Journal of informatics \& mathematical sciences, 7(2), 93-97.

[21] Press, W.H., Flannery, B.P., Teukolsky, S.A., Vetterling, W.T. 1992. Initial Value Problems. Numerical Recipes in FORTRAN: The Art of Scientific Computing, 2nd ed. Cambridge, England: Cambridge University Press, 829.

[22] Dukkipati, R.V. 2008. MATLAB: an introduction with applications. New Age International.

[23] Pratap, R. 2010. Getting started with MATLAB: a quick introduction for scientists and engineers. USA: Oxford University Press.

[24] Feng, G.A.O. 2011. Applications of Matlab in mathematical analysis, Journal of Software, 6(7), 1225-1229.

[25] Wolfram, S. 1984. Computer software in science and mathematics, Scientific American, 251(3), 188-203.

[26] Stanoyevitch, A. 2011. Introduction to Numerical Ordinary and Partial Differential Equations Using MATLAB, John Wiley \& Sons, 72. 